\title{
New directions in cognitive training: on methods, transfer, and application
}

\author{
Torsten Schubert · Tilo Strobach · Julia Karbach
}

Received: 15 September 2014/ Accepted: 24 September 2014/Published online: 11 October 2014

(C) Springer-Verlag Berlin Heidelberg 2014

Research on cognitive interventions and training-induced changes in brain and behavior has been of growing interest in psychology and neuroscience over the last decade (for reviews see Hertzog, Kramer, Wilson, \& Lindenberger, 2009; Lustig, Shah, Seidler, \& Reuter-Lorenz, 2009; Chein \& Morrison, 2010; for meta-analyses see Karbach \& Verhaeghen, 2014; Melby-Lervag \& Hulme, 2013). The interest in these studies is to a large extent related to empirical reports and observations, which suggest that appropriate training interventions can produce broad learning effects and may generalize to other non-trained tasks (e.g., Bergman-Nutley et al., 2011; Green \& Bavelier, 2003; Jaeggi, Buschkuehl, Jonides, \& Perrig, 2008; Klingberg, 2010; Strobach, Frensch, \& Schubert, 2012, and many others). These training and transfer effects are often observed in studies using process-based training; this training is based on the assumption that practice and learning may improve relevant processes in one task situation and the improvement may generalize to another task situation if there is a process overlap between situations (Schmidt \& Bjork, 1992; Taatgen, 2013). This kind of training must be distinguished from (rather traditional) strategy-based training approaches, which usually focus on

T. Schubert $(\bowtie) \cdot$ T. Strobach

Department of Psychology, Humboldt-Universität zu Berlin, Rudower Chaussee 18, 12489 Berlin, Germany

e-mail: torsten.schubert@psychologie.hu-berlin.de

T. Strobach

Department of Psychology, FernUniversität Hagen, 58084 Hagen, Germany

J. Karbach

Department of Educational Science, Saarland University, 66123 Saarbrücken, Germany the training of task-specific procedures and strategies improving the performance in the given task without transferring broadly to other tasks (i.e., Klauer, 1989; Kliegl \& Baltes, 1987; Kliegl, Smith, \& Baltes, 1990; Rebok, Carlson, \& Langbaum, 2007).

In particular, the implications of broad transfer as a result of process-based and other trainings are manifold and have given rise to the increasing interest in current training research. First of all, since many of the reported training effects are related to basic cognitive domains like attention, working memory, executive functions, and intelligence, recent cognitive training studies seem to promise a training-related boost of the cognitive machinery on a broad scale and functioning. Broad training effects have been reported across the whole life span from the young to the elderly brain, although with different degrees of efficiency. This offers even a life-span perspective of the occurrence of training-related boosting effects (e.g., Brehmer et al., 2008, for reviews see Diamond, 2012; Hertzog et al., 2009 and others). Second, a further and more theoretical appeal of this new training research originates from the fact that its findings may challenge two cornerstones of knowledge that guided theoretical and empirical research in experimental psychology and cognitive neuroscience so far: Ad one, for a long time, it was as given that cognitive capabilities usually mature at the end of adolescence because the growth of cognitive functions is due to an increase in cognitive capacity that has finished at that age, and a further remarkable boost of cognitive capacity is not possible. Ad two, if there is cognitive plasticity beyond adolescence then this is either reflected in cognitive improvements due to highly specific learning of task-specific algorithms and knowledge, or in impairments of cognitive functions resulting from an age-related decline. Yet, the results of studies on cognitive 
interventions and training-induced changes in basic cognitive functions like attention, working memory, executive functions, etc. seem to cast doubts on these two cornerstones of psychological knowledge. Last but not least, reports of broad training and transfer effects provide researchers with a new possibility for the application of their findings in everyday-like situations, such as education, clinical contexts, gaming, cognition of the elderly, and others. This interplay may enhance the impact of basic research in cognitive psychology and cognitive neuroscience for societal purposes (Strobach \& Schubert, 2014).

Despite the promising initial findings, there are a number of important questions related to current research on cognitive training (e.g., Karbach \& Schubert, 2013; Shipstead, Redick, \& Engle, 2012). These are questions about the validity and reliability of the findings and about methodological standards in current cognitive training research. Other questions relate to the types of training, which are or are not beneficial for the occurrence of broad transfer effects across tasks and functions. Further questions address the special conditions of the tasks measuring the success of cognitive training, the specific conditions of the training and the trainee, which enable or prevent cognitive training-related plasticity and the sustainability of the changes.

The aim of this current special issue is to provide a broad report on state-of-the-art research on new directions in cognitive training and to advance our understanding of the scope, the possibilities, and the mechanisms of trainingrelated changes in cognitive functions and in the related brain structures. For this purpose, we invited seven reviews and four empirical articles for the present special issue. These articles focus (1) on methodological issues in cognitive training research, (2) on issues of preconditions for the occurrence of training-related transfer effects, and, (3) on possible applications of cognitive training for the improvement of cognitive functions in everyday-like situations.

(1) Methodological issues in cognitive training research. Two review articles (Green, Strobach, \& Schubert, 2014; Noack, Lövden, \& Schmiedek, 2014) address prevailing methodological issues and provide comprehensive methodological guidelines for efficient cognitive training research. As an important step for the further development of cognitive training research, Green et al. (2014) start with an overview of the most important methodological caveats in cognitive training research. These caveats are related to expectation effects of the participants, the occurrence of test-retest effects, the selection of appropriate transfer tasks, the proper selection of control conditions and the allocation of subjects to different experimental conditions. Most researchers in the field of cognitive training research will acknowledge that these issues are most urgent when setting up a cognitive training study. As an example, differences in the expectation of certain training effects by participants in different training groups may have an influence on the final results of a training intervention as had been suggested by Boot and Simons (2012) and others (but see Schubert \& Strobach, 2012; Strobach, Frensch, \& Schubert, 2012). The insufficient control of these possible expectation effects may harm the validity and reliability of the conclusion. Green et al. describe the possible caveats and assess their potential impact on the outcome of studies and show that this impact may be erroneously underestimated but sometimes even overestimated. Most importantly, the authors not only describe possible caveats, but also provide applicable and useful methodological guidelines on how to deal with them. Applying these guidelines will make methodologically valid research possible that is not overcontrolled and too conservative.

The methodological review by Noack et al. (2014) introduces the terms, generality and specificity into the methodological perspective of cognitive training and intervention research, to enhance theory-driven training studies. The term generality refers to the methodological demand to apply test batteries in training studies that allow the analysis of transfer effects with multiple paradigms tapping on a defined cognitive domain. The realization of multiple (instead of single) paradigms allows testing for transfer effects beyond the task-specific skills on a latent construct level. Specificity, on the other hand, requires the realization of transfer tasks and measures that are plausible from a theoretical perspective because of shared processes between training and transfer tasks. From a methodological point of view, the approach requires a careful comparison of the trainee's performance in these especially defined tasks and the performance in tasks that probably do not reveal transfer because of lacking process overlap. The authors clarify that most training studies are not yet constructed in a way that is appropriate to test for generality and specificity of a proposed training intervention. Creating studies which focus on the generality and specificity issue of training effects should be an important future research direction and will require increased effort for the realization of training studies (see also Shipstead et al., 2012).

The studies of Green et al. (2014) and Noack et al. (2014) show that research on cognitive training interventions has started to outgrow its childhood. The initial phase of research on cognitive training effects, which was characterized by enthusiastic estimations (sometime overestimations) of the possibilities of training research has been cooling down and a phase has been evolving in which a rational assessment of possibilities and limitations takes place; this relates to a critical specification of paradigms and methodological guidelines for efficient and valid cognitive training research. 
The impression that research on cognitive training is going to outgrow childhood is also underlined by the occurrence of multimodal cognitive training studies. Most importantly, numerous studies do not focus exclusively on the behavioral consequences of cognitive trainings anymore, but investigate the accompanying changes in the brain (e.g., Belleville \& Bherer, 2012; Dahlin, Neely, Larsson, Bäckman, \& Nyberg, 2008; Nyberg et al., 2003; for a review, see Klingberg, 2010, and many others). This neural perspective is guided by an underlying assumption about plasticity, which influences the studies of many researchers in the field, more or less implicitly (Lövdén, Bäckman, \& Lindenberger, 2010). According to that assumption, permanent cognitive and neural changes may emerge if a certain (brain) mechanism is required by a given task in a manner that exceeds the possible available resources of the organism for a sufficiently long period. In that case changes may occur which result in permanent changes of the cognitive and brain system and, consequently, in cognitive and brain plasticity (Lövden, Wenger, Mårtensson, Lindenberger, \& Bäckman, 2013).

Brehmer, Kalpouzus, Wenger and Lövden (2014) review findings about emerging training-related brain changes, especially in the older brain, after strategy-based and process-based cognitive training as well as physical exercise training. The authors show that these trainings may lead to plastic changes on three different levels in the elderly: On the behavioral level, the level of functional brain activations and the level of structural brain changes (for similar findings in younger adults see Dahlin et al., 2008; Klingberg, 2010, and others). A particularly important question with respect to changes in functional brain activation relates to the direction of the brain changes: does training lead to increases or decreases of brain activations as a result of training a certain function? Brehmer et al. (2014) show that both outcomes are possible. Whereas the decrease in brain activation along with an improvement of the function indicates increased efficiency, increases in brain activation may indicate the recruitment of additional brain regions and mechanisms that are required by the executed function. An important issue relates to the special relationship between functional and structural changes. While it seems necessary that functional brain activity changes to precede a possible structural brain change, not every training-related increase or decrease of functional activation necessarily results in structural brain changes. The authors discuss the possible factors that may influence the probability that a particular type of training intervention leads to structural changes. Among them, the authors highlight the factors like training length and intensity, task difficulty, type of training, and baseline performance of individual subjects. Future studies on neuroplasticity should apply multimodal research approaches combining data on training-related functional and structural brain changes. Furthermore, these studies should focus on individual differences, on the relationship between brain changes and training gains, and on the time course of brain changes (Brehmer et al., 2014).

(2) Preconditions for the occurrence of training-related transfer effects. An important set of questions in current training research relates to specific characteristics of the training paradigms, the training types and the individual trainees that might promote or prevent the occurrence of transfer effects. A number of articles (Bürki, Ludwig, Chicherios, \& de Ribaupierre, 2014; Colzato, van den Wildenberg, \& Hommel, 2011; Strobach, Salminen, Karbach \& Schubert, 2014; von Bastian \& Oberauer, 2014), discuss these characteristics. For instance, von Bastian and Oberauer (2014) provide a report in which they review selected factors modulating the effects of training on working memory and reasoning processes. The authors propose that two general mechanisms are essential for beneficial training effects on working memory and reasoning performance: Enhanced capacity and enhanced efficiency of working memory processes. However, they also acknowledge that findings of transfer effects after working memory training are still controversial and sometimes even contradictory. As possible causes for this mixed evidence, von Bastian and Oberauer discuss the potential impact of training features (such as the type of training paradigm, the training intensity, duration, and adaptivity of training difficulty) and individual factors (such as cognitive abilities before training, age, genetic predispositions, motivational factors, and personality traits).

The empirical study of Bürki et al. (2014) highlights the necessity to consider individual differences to predict success of training-related near and far transfer effects. The authors argue that individual differences in training-related gains and their dynamics can be crucial information for the successful prediction of learning and transfer effects. In particular, they propose to examine the relationship between the growth of individual performance across training sessions and the amount of working memory benefits with latent growth curve modeling (LGCM). They illustrate the concept with a study in which they investigated the individual trajectories of training performances across 10 days of working memory training in younger and older adults $(N=128)$. The authors found that younger adults started the training at a higher performance level and improved faster during training, resulting in a magnification of age differences. Individual differences in fluid intelligence explained individual differences in the initial training performance, independent of the age group. On the other hand, participants' age predicted individual differences in training progression beyond individual differences 
in fluid intelligence. The findings of the study emphasize that data on individual differences in training-induced performance gains (for example, as measured by LGCM), should play an important role when developing tailored training interventions.

A factor often assumed to affect the occurrence of training-related transfer effects is the genetic predisposition of participants. Colzato et al. (2014) show this for the case of transfer effects on executive functions, such as cognitive flexibility, which occurred after action video game training (see also Strobach et al., 2012). The impact of the genetic predisposition was analyzed for the enzyme COMT $\mathrm{Val}^{158} \mathrm{Met}$, which regulates the dopamine level in prefrontal and other cortical as well as subcortical regions (Bilder, Volavka, Lachman, \& Grace, 2004). Individuals with a predisposition for low cortical dopamine levels (Val/ Val-carriers) demonstrated an improved performance in a task-switching transfer test after action video game training compared to individuals with a predisposition for high prefrontal dopamine levels (Met-carriers). This is consistent with the assumptions of Bilder et al. (2004) and others that cognitive flexibility might benefit from relatively low prefrontal dopamine levels, which allows the flexible updating of processed representations (but see Braver, Barch, \& Cohen, 1997). This result demonstrates that transfer effects in untrained tasks are related to the genetic predisposition of trainees and confirms observations of other training studies (e.g., working memory training; Bäckman \& Nyberg, 2013; Rieckmann et al., 2012).

The occurrence of training-related transfer effects may also be related to the overlap of processing mechanisms in training tasks and transfer tasks (Chein \& Morrison, 2010; Dahlin, Nyberg, Bäckman, \& Neely, 2008; Schmidt \& Bjork, 1992). Strobach et al. (2014) follow a strict processbased perspective to explain the occurrence of trainingrelated transfer effects in dual-task situations. They first provide an overview of transfer effects on the executive functions such as shifting, inhibition, and updating (cf. Miyake, Friedman, Emerson, Witzki, \& Howerter, 2000). In addition, they review recent findings indicating reliable transfer of extended dual-task training to new dual-task situations (see also Liepelt, Strobach, Frensch, \& Schubert, 2011) and propose a theoretical model to explain these effects. According to this Efficient Task Initiation model (ETI), dual-task performance improves with dual-task practice because of an efficient and simultaneous instantiation of relevant task information for two component tasks in working memory at the onset of a dual-task trial. Training-related transfer to other dual-task situations may occur if the training requires the simultaneous instantiation of two task sets at the same time. Accordingly, an efficient instantiation is realized after dual-task training, but not after single-task training without conjoint activation of the two task sets. As a crucial assumption, dual-task transfer effects should also occur after special types of single-task training, which, as a result of a mixed task presentation during training, require the simultaneous activation of two task sets. The authors provide evidence in favor of their model by reporting the findings of a corresponding dualtask training experiment. The successful prediction of transfer effects by the ETI model shows that process-oriented models of executive functions may be successfully informed by cognitive training studies and vice versa.

(3) Applications of cognitive training in everyday-like situations. Cognitive training studies are often confronted with the expectation that training should lead to changes in skills that improve cognition and behavior in everyday-like situations. Several articles (Spruit, Band, Hamming, \& Ridderinkhof, 2014; Hering, Rendell, Rose, Spitzspahn, \& Kliegel, 2014; Klingberg \& Bergman-Nutley, 2014; Titz \& Karbach, 2014) address this perspective in different domains. Titz and Karbach (2014) focus on the field of education which has a tremendous potential for the possible application of cognitive training research. The authors discuss the question to which extent cognitive training may benefit academic abilities and skills in younger children up to adolescence. Although findings are still far from being conclusive and, in some cases, not unequivocal, the existing evidence already allows the conclusion that certain cognitive trainings can indeed influence scholastic skills beneficially. This evidence originates mainly from studies of process-based complex working memory trainings and is particularly pronounced in the domain of reading and language skills in groups of both healthy children as well as children suffering from cognitive deficits (Karbach, Strobach, \& Schubert, 2014). On an individual level, one may assume the training to be more beneficial for individuals already exhibiting high-level cognitive performance before the training (magnification effect) because they have more cognitive resources to implement new strategies and abilities. On the other hand, one may also assume that the training may be more beneficial for low-performing children because they have more room for improvement (compensation effect). The review by Titz and Karbach reports findings from previous studies showing that process-based trainings of working memory processes and other executive functions often result in larger effects for low-performing individuals (compensation effect).

Results of Klingberg and Bergman-Nutley (2014) confirm that working memory training can particularly improve the performance of children with working memory deficits in arithmetic and other everyday-like activities like remembering and following classroom instructions. An important characteristic of Klingberg and Bergman-Nutley's study (compared to many others) is that the authors investigated training effects with a working memory 
training battery (i.e., Cogmed) in a large sample of 176 children (7-15 years), who suffered from working memory deficits, over 5 weeks of training. The data of these children were compared with the data of 304 normally developing children (7-15 years) who did not train, but performed the same transfer tasks at the beginning and at the end of the 5 week period with weekly transfer tests in between. Results showed that children with working memory deficits improved more on the transfer tasks than normally developing children. The authors acknowledge that the effect sizes were small for the mathematics test and up to moderate and large for the working memory tests. However, these effect sizes should be compared with effects sizes of other types of interventions, including those affecting the underlying brain physiology. Accordingly, the authors noted that the training-induced improvement in mathematics in their study (effect size $=0.2$ standard deviations, or $8 \%$ ) is comparable with the effects of medication for depression, Alzheimer's disease, and ADHD (effect sizes 0.2-0.3 standard deviations). The findings of Klingberg and Bergman-Nutley are, therefore, highly encouraging to the perspectives of training interventions in education research and education itself.

Spruit et al. (2014) focus on laparoscopic surgery to propose a further domain for the application of cognitive training effects. In this minimally invasive surgical technique, operations are performed through small incisions by means of a laparoscope. While this technique is associated with tremendous benefits for the patients when compared to traditional surgical techniques, intensive training is necessary for the surgeons to perform the corresponding motor actions with sufficient precision and efficiency. The authors discuss different findings on training complex procedural, perceptual, and motor skills that are of relevance for laparoscopic surgery. They highlight different training models regarding motor skills and their automatisation as a function of learning and training. Based on these theoretical frameworks and empirical findings, they propose a set of recommendations for laparoscopic motor skill training, including goal setting, adaptivity and variability of the training, as well as elements of deliberate practice. These recommendations in an applied professional context are consistent with recommendations for training procedures in more traditional areas of cognitive training like working memory training and training of executive functions (von Bastian \& Oberauer, 2014; Strobach et al., 2014).

Cognitive interventions designed for individuals suffering from memory loss or deficits in executive functions are a further domain for the application of cognitive training. Deficits in these functions are strongly evident in prospective memory, i.e., the memory for actions which have to be performed in the future at certain points in time or under certain conditions. Hering et al. (2014) discuss the possibilities to improve prospective memory functions, particularly in the elderly, who suffer most from an impairment of prospective memory. The authors review studies focusing on strategy-based and process-based training procedures. Strategy-based trainings focus on learning and practicing special procedures of handling the specific steps and phases during prospective memory, like the creation of informative markers, when to remember or how to remember. In contrast, process-based trainings of prospective memory are based on the assumption that processes, like updating of working memory and executive control, are relevant during prospective memory and can benefit from corresponding training-related improvements. Indeed, first studies show positive effects of process-based trainings on several aspects of prospective memory, which increases the range of applicability of cognitive training for improving everyday activities. The authors conclude that a combination of strategy-based trainings and general boosts in cognitive functioning due to process-based training of working memory is most promising for improving prospective memory in the elderly. The proposed perspective of a tailor-made combination of task-specific mechanisms with a general boost of the underlying cognitive machinery represents an important future direction for cognitive training research.

Altogether, current research on cognitive training represents a highly promising area for psychology, neuroscience, and related disciplines as well as for the application of their findings in real-world scenarios. The articles in the current special issue show that research on cognitive training is evolving and that a set of new methodological guidelines facilitates the design of valid and efficient training studies. A number of important theoretical issues regarding the processes and mechanisms underlying training and transfer effects are raised by the findings of current cognitive training studies; these include the characteristics of the training, the trained task, the transfer tasks, and the trainees. Finally, a great deal of current research on cognitive training is related to the expectations regarding the applicability of the findings in real-world scenarios and activities of daily life. Although the studies in the current special issue do not allow final conclusions as to whether these expectations can be fulfilled, they draw a rather encouraging picture regarding the potential of cognitive training for improving mental functions in a number of everyday-like situations. This relates to education in school and professional contexts, clinical situations, cognition of the elderly, and others. However, the current studies also emphasize the need to further investigate cognitive training from both a theoretical perspective and an applied perspective to identify the mechanisms mediating training and transfer effects. 


\section{References}

Bäckman, L., \& Nyberg, L. (2013). Dopamine and training-related working-memory improvement. Neuroscience and Biobehavioral Reviews, 37, 2209-2219. doi:10.1016/j.neubiorev.2013.01. 014.

Belleville, S., \& Bherer, L. (2012). Biomarkers of cognitive training effects in aging. Current Translational Geriatrics and Experimental Gerontology Reports, 1, 104-110. doi:10.1007/s13670012-0014-5.

Bergman-Nutley, S., Söderqvist, S., Bryde, S., Thorell, L. B., Humphreys, K., \& Klingberg, T. (2011). Gains in fluid intelligence after training non-verbal reasoning in 4-year-old children: a controlled, randomized study. Developmental Science, 14(3), 591-601. doi:10.1111/j.1467-7687.2010.01022.x.

Bilder, R. M., Volavka, J., Lachman, H. M., \& Grace, A. A. (2004). The catechol- $O$-methyltransferase polymorphism: relations to the tonic-phasic dopamine hypothesis and neuropsychiatric phenotypes. Neuropsychopharmacology, 29, 1943-1961.

Boot, W. R., \& Simons, D. J. (2012). Advances in video game methods and reporting practices (but still room for improvement): a commentary on Strobach, Frensch, and Schubert (2012). Acta Psychologica, 141(2), 276-277. doi:10.1016/j. actpsy.2012.06.011.

Braver, T., Barch, D. M., \& Cohen, J. D. (1997). Cognition and control in schizophrenia: a computational model of dopamine and prefrontal function. Biological Psychiatry, 46, 312-328.

Brehmer, Y., Kalpouzos, G., Wenger, E., \& Lövden, M. (2014). Plasticity of brain and cognition in older adults. Psychological Research (this issue).

Brehmer, Y., Li, S. C., Straube, B., Stoll, G., von Oertzen, T., Müller, V., et al. (2008). Comparing memory skill maintenance across the life span: preservation in adults, increase in children. Psychology and Aging, 23, 227-238. doi:10.1037/0882-7974. 23.2.227.

Bürki, C., Ludwig, C., Chicherio, C., \& de Ribaupierre, A. (2014). Individual differences in cognitive plasticity: an investigation of training curves in younger and older adults. Psychological Research (this issue).

Chein, J. M., \& Morrison, A. B. (2010). Expanding the mind's workspace: training and transfer effects with a complex working memory span task. Psychonomic Bulletin \& Review, 17(2), 193-199.

Colzato, L., van den Wildenberg, W., \& Hommel, B. (2014). Cognitive control and the COMT Val158Met polymorphism: genetic modulation of videogame training and transfer to taskswitching efficiency. Psychological Research (this issue).

Dahlin, E., Neely, A. S., Larsson, A., Bäckman, L., \& Nyberg, L. (2008a). Transfer of learning after updating training mediated by the striatum. Science, 320, 1510-1512. doi:10.1126/science. 1155466.

Dahlin, E., Nyberg, L., Bäckman, L., \& Neely, A. S. (2008b). Plasticity of executive functioning in young and older adults: immediate training gains, transfer, and long-term maintenance. Psychology and Aging, 23(4), 720-730.

Diamond, A. (2012). Activities and programs that improve children's executive functions. Current Directions in Psychological Science, 21, 335-341.

Green, C. S., \& Bavelier, D. (2003). Action video game modifies visual selective attention. Nature, 423, 534-537.

Green, C. S., Strobach, T., \& Schubert, T. (2014). On Methodological standards in training and transfer experiments. Psychological Research (this issue).

Hering, A., Rendell, P., Rose, N., Schnitzspahn, K., \& Kliegel, M. (2014). Prospective memory training in older adults and its relevance for successful aging. Psychological Research (this issue).

Hertzog, C., Kramer, A. F., Wilson, R. S., \& Lindenberger, U. (2009). Enrichment effects on adult cognitive development. Psychological Science in the Public Interest, 9, 1-65.

Jaeggi, S. M., Buschkuehl, M., Jonides, J., \& Perrig, W. J. (2008). Improving fluid intelligence with training on working memory. Proceedings of the National Academy of Sciences, 105(19), 6829-6833.

Karbach, J., \& Schubert, T. (2013). Training-induced cognitive and neural plasticity. Frontiers of Human Neuroscience, 7, 48.

Karbach, J., Strobach, T., \& Schubert, T. (2014). Adaptive workingmemory training benefits reading, but not mathematics in middle childhood. Child Neuropsychology. Advance Online Publication. doi:10.1080/09297049.2014.899336.

Karbach, J., \& Verhaeghen, P. (2014). Making working memory work: a meta-analysis of executive control and working memory training in older adults. Psychological Science (to appear).

Klauer, K. J. (1989). Handbuch kognitives training. Göttingen: Hogrefe.

Kliegl, R., \& Baltes, P. B. (1987). Theory-guided analysis of mechanisms of development and aging mechanisms through testing-the-limits and research on expertise. In C. Schooler \& K. W. Schaie (Eds.), Cognitive functioning and social structure over the life course (pp. 95-119). Norwood, NJ: Ablex.

Kliegl, R., Smith, J., \& Baltes, P. B. (1990). On the locus and process of magnification of age differences during mnemonic training. Developmental Psychology, 26, 894-904.

Klingberg, T. (2010). Training and plasticity of working memory. Trends in Cognitive Sciences, 14(7), 317-324. doi:10.1016/j.tics. 2010.05.002.

Klingberg, T., \& Bergman-Nutley, S. (2014). Effect of working memory training on working memory, arithmetics, and following instructions. Psychological Research (2014).

Liepelt, R., Strobach, T., Frensch, P., \& Schubert, T. (2011). Improved intertask coordination after extensive dual-task practice. The Quarterly Journal of Experimental Psychology, 64(7), 1251-1272.

Lövden, M., Bäckman, L., Lindenberger, U., Schaefer, S., \& Schmiedek, F. (2010). A theoretical framework for the study of adult cognitive plasticity. Psychological Bulletin, 136(4), 659-676. doi:10.1037/a0020080.

Lövden, M., Wenger, E., Mårtensson, J., Lindenberger, U., \& Bäckman, L. (2013). Structural brain plasticity in adult learning and development. Neuroscience and Biobehavioral Reviews, 37, 2296-2310.

Lustig, C., Shah, P., Seidler, R., \& Reuter-Lorenz, P. A. (2009). Aging, training, and the brain: a review and future directions. Neuropsychology Review, 19, 504-522. doi:10.1007/s11065009-9119-9.

Melby-Lervag, M., \& Hulme, C. (2013). Is working memory effective? A meta-analytic review. Developmental Psychology, 49(2), 270-291. doi:10.1037/a0028228.

Miyake, A., Friedman, N. P., Emerson, M. J., Witzki, A. H., \& Howerter, A. (2000). The unity and diversity of executive functions and their contributions to complex "frontal lobe" tasks: a latent variable analysis. Cognitive Psychology, 41, 49-100.

Noack, H., Lövden, M., \& Schmiedek, F. (2014). On the validity and generality of transfer effects in cognitive training research. Psychological Research (this issue).

Nyberg, L., Sandblom, J., Jones, S., Neely, A. S., Petersson, K. M., Ingvar, M., et al. (2003). Neural correlates of training-related memory improvement in adulthood and aging. Proceedings of the National Academy of Sciences of the United States of America, 100, 13728-13733. 
Rebok, G. W., Carlson, M. C., \& Langbaum, J. B. (2007). Training and maintaining memory abilities in healthy older adults: traditional and novel approaches. The Journals of Gerontology Series B: Psychological Sciences and Social Sciences, 62, 53-61.

Rieckmann, A., Karlsson, S., Karlsson, P., Brehmer, Y., Fischer, H., Farde, L., et al. (2012). Dopamine D1 receptor associations within and between dopaminergic pathways in younger and elderly adults: links to cognitive performance. Cerebral Cortex, 21, 2023-2032. doi:10.1093/cercor/bhq266.

Schmidt, R. A., \& Bjork, R. A. (1992). New conceptualizations of practice: common principles suggest new concepts for training. Psychological Science, 3(4), 207-217.

Schubert, T., \& Strobach, T. (2012). Video game experience and optimized executive control skills—on false positives and false negatives: Reply to Boot \& Simons (2012). Acta Psychologica, 141(2), 278-280.

Shipstead, Z., Redick, T. S., \& Engle, R. W. (2012). Is working memory training effective? Psychonomic Bulletin, 138(4), 628-654. doi:10.1037/a0027473.

Spruit, E., Band, G., Hamming, J., \& Ridderinkhof, R. (2014). Optimal training design for procedural motor skills: a review and application to laparoscopic surgery. Psychological Research. doi:10.1007/s00426-013-0525-5 (this issue).

Strobach, T., Frensch, P. A., \& Schubert, T. (2012). Video game practice optimizes executive control skills in dual-task and task switching situations. Acta Psychologica, 140(1), 13-24.

Strobach, T., Salminen, T., Karbach, J., \& Schubert, T. (2014). Practice-related optimization and transfer of executive functions: a general review and a specific realization of their mechanisms in dual tasks. Psychological Research (this issue).

Strobach, T., \& Schubert, T. (2014). Positive consequences of actionvideo game experience on human cognition: potential benefits on a societal level. In K. K. Mak (Ed.), Epidemiology of online game addiction. OMICS Group.

Taatgen, N. A. (2013). The nature and transfer of cognitive skills. Psychological Review, 120(3), 439-471.

Titz, C., \& Karbach, J. (2014). Working memory and executive functions: Effects of training on academic achievement. Psychological Research (this issue).

von Bastian, C.C., \& Oberauer, K. (2014). Effects and mechanisms of working memory training: a review. Psychological Research (this issue). 